\title{
Synthesis of New Brassinosteroid Biosynthesis Inhibitor with Coumarin Moiety as a Fluorescent Probe
}

\author{
Tomoki Hoshi, Tadashi Matsumoto, Kazuhiro Yamada, Yuko Yoshizawa, and Keimei Oh
}

\begin{abstract}
Plants responses to internal signals and environmental stimuli are regulated by a complex mechanism of signal transduction networks. Brassinosteroids (BRs) are important signal mediators which play significant role in plant development and defense responses to environmental stress. To explore the biological functions of BRs biosynthetic pathways, we conducted a systematic search for specific inhibitors of BRs biosynthesis. In the present work, we report the synthesis of a novel triazole derivative with coumarin moiety (YCZ-FL) as a fluorescent probe. The fluorescence properties and the biological activity of YCZ-FL on BRs biosynthesis inhibition were investigated. We found that YCZ-FL exhibits highly fluorescence intensity with a maximum excitation wavelength at $336 \mathrm{~nm}$, and maximum emission wavelength at $442 \mathrm{~nm}$, respectively. YCZ-FL inhibits stem elongation of Arabidopsis seedlings grown in the darkness with an $\mathrm{IC}_{50}$ approximately 1.56 $\pm 0.05 \mu \mathrm{M}$. To distinguish the primary site of actions on biosynthesis of GA and BRs, effect of co-application of GA (1 $\mu \mathrm{M})$ and/or BL $(10 \mathrm{nM})$ on YCZ-FL treated Arabidopsis seedlings were evaluated. We found that co-application of $B L$ reversed the inhibition of stem elongation treated by while GA did not.
\end{abstract}

Index Terms-Brassinosteroid biosynthesis inhibitor, fluorescent labeling probe, plant growth regulators.

\section{INTRODUCTION}

Brassinosteroids (BRs) are important signal mediators that involve in wide array of physiological process through activation gene expression in plant growth and development. Molecular genetic analysis established the role of BRs as endogenous plant hormones with well-defined functions, including stem elongation, pollen tube growth, leaf bending, leaf unrolling, root inhibition, proton pump activation [1]-[5]. Because BRs control several important agronomic traits such as flowering, plant architecture, seed yield, and stress tolerance, [2], [3] efforts have been made to control BRs levels in plant tissues using genetic approaches.

Overexpression of DWARF4, an enzyme that catalyses a rate-limiting step in BRs biosynthesis, enhances plant growth and seed yield in Arabidopsis. [6] Mutants that disrupt enzymes of BRs biosynthesis in Arabidopsis, rice, tomato and pea display a common features of reduced plant height phenotypes. [7]-[10] Available evidence indicates that mutations in BRs biosynthesis may be a means to improve biomass production. [11] In this context, manipulating BRs

Manuscript received December 1, 2013; revised February 25, 2014. This work was supported by the president project of Akita Prefectural University.

The authors are with the Department of Biotechnology, Faculty of Bioresource Sciences, Akita Prefectural University, 241-438, Shimoshinjo Nakano, Akita 010-0195, Japan (e-mail: jmwang@akita-pu.ac.jp). content in plant tissues may have remarkable effects on plant growth. Consequently, knockout mutations of BRs biosynthesis may be useful for breeding dwarf plants for agricultural applications.

An alternative way to manipulate the BRs levels in plant tissues is the use of specific inhibitors targeting BRs biosynthesis. Use of inhibitors to control BRs levels in plant tissues is thought to have advantages over using genetic mutants. First of all, application of bio-active small molecules has played a crucial role in identifying novel components involved in plant hormone signal transduction pathways. [12] Secondly, inhibitors can be used at different stages of plant growth and development. Additionally, specific inhibitors of BRs biosynthesis can be applied to different plant species with great ease. And most importantly, use of small molecules allows bypassing the genetic problems of lethality and redundancy due to the target site of small molecules are proteins. Against this background, we carried out a systematic search for specific inhibitors of BRs biosynthesis in consequence of the increasing needs of developing new small molecules for dissecting the mechanism of plant hormone signalling.

Fluorescent labeled small molecules are powerful tools for cell biologists. They provide high sensitivity for monitoring complex cellular processes. It has been demonstrated that the fluorescently labeled inhibitors can be used as a probe for functional analysis the target proteins [13].

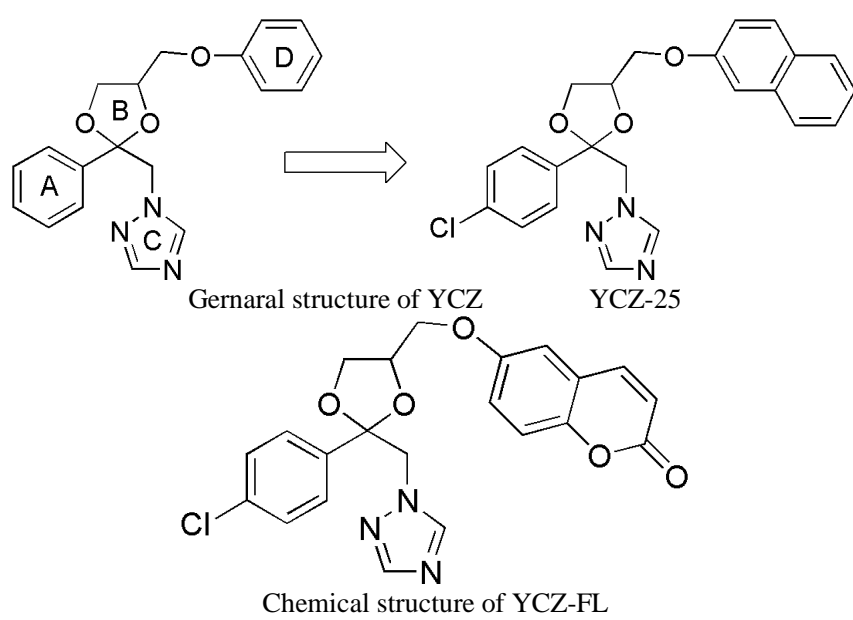

Fig. 1. Chemical structures of YCZs.

Our research interest is the design and synthesis of novel plant hormone biosynthesis inhibitors. [14]-[20] In the course of work, we carried out a bio-rational approach by using ketoconazole as a molecular scaffold, which lead to the discovery of a new series of BRs biosynthesis inhibitors. (YCZ-series, chemical structure shown in Fig. 1) [19]. 
Structure-activity relationship studies indicated that the introduction of a mono substitution of a chlorine atom at position 4 or double substitutions of chlorine atoms at positions 2 and 4 on ring A (Fig. 1) exhibited potent inhibition on BR biosynthesis. [21] We also found that substitutions on ring $\mathrm{D}$ (as shown in Fig. 1) dramatically affected the inhibitory potency against BRs biosynthesis of this synthetic series [22]. Importantly, we found that analogue of YCZ-25 which has a naphthalene moiety at ring $\mathrm{D}$ exhibit potent inhibitory activity on retardation stem elongation of seedlings with $\mathrm{IC}_{50}$ value approximately $0.41 \pm 0.08 \mu \mathrm{M}$ [21].

The above observations provide information that allows us to design new fluorescent labelled BRs biosynthesis inhibitors which is useful for investigation the action mechanism of
YCZ-series on BRs biosynthesis at cell level. Based on these reasons, we conducted a chemical approach by synthesis fluorescence labeled YCZ.

YCZ-25 which has a naphthalene moiety exhibited potent inhibitory activity against BRs biosynthesis. [21] On the other hand, coumarin which exhibits strong fluorescent properties can thus be introduced to the YCZ as a candidate of fluorescent probe. Thus, we designed new fluorescent labeled BRs biosynthesis inhibitor by introducing 6-hydroxycoumarin into the YCZ skeleton (As shown in Fig. 1). In the present work, we report the synthesis and biological evaluation of new BRs biosynthesis inhibitors of YCZ-FL with coumarin moiety.<smiles>O=C(Cn1cncn1)c1ccc(Cl)cc1</smiles>

1

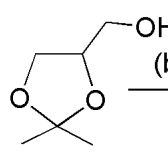

3

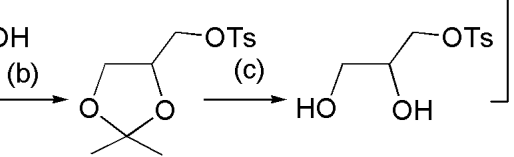

4

5

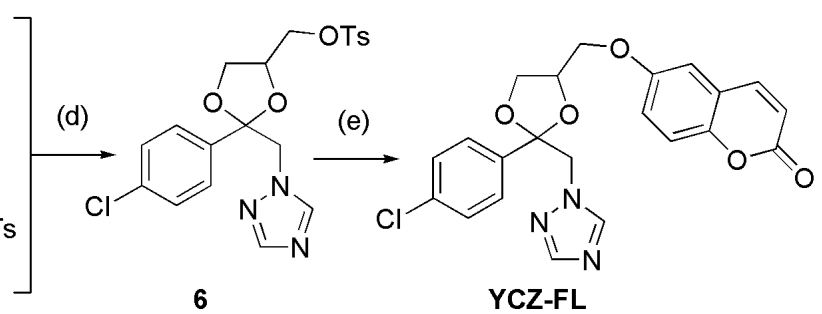

Reagents and conditions: (a) 1,2,4-triazole, triethylamine, DMF, $-10^{\circ} \mathrm{C}, 1$ h, r.t., 3 h; (b) TsCl, pyridine, $0^{\circ} \mathrm{C}$, acetone; (c) $\mathrm{HCl}$, Reflux, 6 h; (d) 3 equiv. TfOH, toluene, r.t., $60 \mathrm{~h}$; and (e) 6-hydroxycoumarin, $\mathrm{KOH}, \mathrm{DMF}, 50^{\circ} \mathrm{C}, 12 \mathrm{~h}$.

Scheme 1. The chemical synthesis of target compounds.

\section{MATERIALS AND MEthods}

\section{A. General}

Chemicals for synthesis were purchased from Kanto Chemicals Co., Ltd (Tokyo, Japan) and Tokyo Kasei Co., Ltd (Tokyo, Japan). Melting points (mp) were determined using a Yanako melting point apparatus. ${ }^{1} \mathrm{H}$ NMR spectra were recorded with a JEOL ECP-400 spectrometer, with chemical shifts expressed in ppm downfield from tetramethylsilane as an internal standard. High resolution mass spectra by electrospray ionization Fourier transform ion cyclotron resonance (ESI-FTICR) was taken using an Exactive MS System (Thermo Fisher Scientific, USA).

\section{B. Chemical Synthesis}

1-(4-chlorophenyl)-2-[1,2,4]-triazol-4-yl-ethanone (2). 2 was prepared using 4-chlorophenacyl bromide as the starting material, as described previously [21]. ${ }^{1} \mathrm{H}-\mathrm{NMR}(400 \mathrm{MHz}$, acetone- $\left.\mathrm{d}_{6}\right) \delta: 5.93(\mathrm{~s}, 2 \mathrm{H}), 7.64-7.68(\mathrm{~m}, 2 \mathrm{H}), 8.10-8.14(\mathrm{~m}$, $2 \mathrm{H}), 8.35(\mathrm{~s}, 2 \mathrm{H})$.

2,2-dimethyl-4-(4-methylbenzene-sulfonate)-1,3-dioxolane-4-methanol (4). Preparation of compound 4 was according to a method as we described previously, [21] ${ }^{1} \mathrm{H}-\mathrm{NMR}\left(400 \mathrm{MHz}, \mathrm{CDCl}_{3}\right.$ ), $\delta: 1.32$ (s, 3H), 1.34 (s, 3H), $2.46(\mathrm{~s}, 3 \mathrm{H}), 3.77(\mathrm{dd}, \mathrm{J}=4.9,9.0 \mathrm{~Hz}, 1 \mathrm{H}), 3.95-4.06(\mathrm{~m}, 3 \mathrm{H})$, 4.25-4.31 (m, 1H), $7.35(\mathrm{~d}, \mathrm{~J}=8.1 \mathrm{~Hz}, 2 \mathrm{H}), 7.80(\mathrm{~d}, \mathrm{~J}=8.4 \mathrm{~Hz}$, $2 \mathrm{H})$.

1-tosyloxy-2, 3-propanediol (5). Preparation of compound 5 was according to a method as we described previously, [21] ${ }^{1} \mathrm{H}-\mathrm{NMR}$ (400 MHz, $\mathrm{CDCl}_{3}$ ), $\delta: 2.46$ (s, 3H), 3.63 (dd, J = 5.1,
$11.4 \mathrm{~Hz}, 1 \mathrm{H}), 3.71(\mathrm{dd}, \mathrm{J}=4.2,11.5 \mathrm{~Hz}, 1 \mathrm{H}), 3.92-3.99(\mathrm{~m}$, $1 \mathrm{H}), 4.06-4.13(\mathrm{~m}, 2 \mathrm{H}), 7.37(\mathrm{~d}, \mathrm{~J}=6.6 \mathrm{~Hz}, 2 \mathrm{H})$, and $7.81(\mathrm{~d}$, $\mathrm{J}=6.6 \mathrm{~Hz}, 2 \mathrm{H})$.

2RS, 4RS-toluene-4-sulfonic acid 2-phenyl-2-1,2,4triazol-1-ylmethyl-1,3-dioxolan-4-ylmethyl ester (6). Preparation of compound $\mathbf{6}$ is based on a method as we reported previously. [21] ${ }^{1} \mathrm{H}-\mathrm{NMR}$ (400 $\left.\mathrm{MHz}, \mathrm{CD}_{3} \mathrm{OD}\right) \delta$ : $2.36(\mathrm{~s}, 3 \mathrm{H}), 2.46(\mathrm{~s}, 3 \mathrm{H}), 3.62(\mathrm{dd}, \mathrm{J}=5.1,8.4 \mathrm{~Hz}, 1 \mathrm{H})$, $3.73-3.80(\mathrm{~m}, 2 \mathrm{H}), 3.94-3.97(\mathrm{~m}, 1 \mathrm{H}), 4.20-4.22(\mathrm{~m}, 1 \mathrm{H})$, $4.77(\mathrm{~s}, 2 \mathrm{H}), 7.22-7.24(\mathrm{~m}, 2 \mathrm{H}), 7.41-7.51(\mathrm{~m}, 6 \mathrm{H}), 7.69-7.71$ $(\mathrm{m}, 2 \mathrm{H}), 7.76-7.78(\mathrm{~m}, 2 \mathrm{H}), 8.50(\mathrm{~s}, 1 \mathrm{H})$, and $9.29(\mathrm{~s}, 1 \mathrm{H})$.

6-[2-(4-chlorophenyl)-2-[1,2,4]triazol-1-ylmethyl-[1,3] dioxolan-4-ylmethoxy]coumarin (YCZ-FL). Potassium hydroxide $(160 \mathrm{mg}, 2.8 \mathrm{mmol})$ was added to a solution of tosylate 6 (485 mg, $0.78 \mathrm{mmol})$ and 6-hydroxycoumarin (117 $\mathrm{mg}, 0.72 \mathrm{mmol})$ in dry DMF $(5 \mathrm{~mL})$, and the reaction mixture was heated at $50^{\circ} \mathrm{C}$ overnight. After cooling to room temperature, the reaction mixture was diluted with water (20 $\mathrm{mL})$ and EtOAc $(20 \mathrm{~mL})$, and the organic phase was separated. The aqueous phase was extracted with EtOAc $(3 \times 20 \mathrm{~mL})$. All of the organic layers were combined, washed with brine (20 mL), dried over anhydrous sodium sulfate, filtered, concentrated, and purified by flash chromatography on silica gel (EtOAc/hexanes =1:1), affording $41 \mathrm{mg}$ target compound (11.4\% \% yield), ${ }^{1} \mathrm{H}-\mathrm{NMR} \quad\left(400 \mathrm{MHz}, \quad\right.$ Acetone- $\left.\mathrm{D}_{6}\right)$, $3.78-3.98(\mathrm{~m}, 4 \mathrm{H}), 4.41-4.47(\mathrm{~m}, 1 \mathrm{H}), 4.63(\mathrm{~s}, 2 \mathrm{H}), 6.40(\mathrm{~d}$, $\mathrm{J}=9.6,1 \mathrm{H}), 7.18-7.28(\mathrm{~m}, 3 \mathrm{H}), 7.413(\mathrm{dt}, \mathrm{J} 1=8.7, \mathrm{~J} 2=2.3,2 \mathrm{H})$, 7.56(dt, J1=8.2, J2=2.8, 2H), 7.78(s, 1H), 7.923(d, J=9.6, $1 \mathrm{H}), \quad 8.31(\mathrm{~s}, \quad 1 \mathrm{H})$ The HRMS-ESI calculated for 
$\mathrm{C}_{22} \mathrm{H}_{18} \mathrm{~N}_{3} \mathrm{O}_{5} \mathrm{ClNa}[\mathrm{M}+\mathrm{Na}]+$ was 462.0833 , and 462.0835 was the experimental value.

\section{Bioassay Methods for Evaluation Brassinosteroid Biosynthesis Inhibitors}

Seeds of Arabidopsis (Columbia ecotype) were purchased from Lehle Seeds (Round Rock, TX, U. S. A.). The seeds used for the assay were sterilized in $1 \% \mathrm{NaOCl}$ for $20 \mathrm{~min}$ and washed with sterile distilled water. Seeds were sown on a $1 \%$ solidified agar medium containing half Murashige and Skoog salt in Petri dishes with or without chemicals. Petri dishes were wrapped in four layers of aluminum foil. Plants were grown in the darkness conditions at $22{ }^{\circ} \mathrm{C}$ in a growth chamber. The biological activities of the test compounds were measured 5 days after sowing the seeds. The hypocotyl length of 15-20 Arabidopsis seedlings was measured with a ruler, and each experiment was repeated at least two times.

\section{Determination the Fluorescent Spectra of YCZ-FL}

Fluorescent spectra of YCZ-FL were measured using a F-4500 Fluorescence Spectrophotometer (Hitachi Co. Ltd., Tokyo, Japan) For each data point 10 measurements were taken with the exciting slit and emission slit setting at 10 and $10 \mathrm{~nm}$, respectively. Samples dissolved in the $\mathrm{H}_{2} \mathrm{O}$ containing $0.1 \%$ DMSO were used.

\section{RESULTS AND DISCUSSION}

\section{A. Chemistry}

Methods for preparation of YCZ-FL are according to a synthetic route (Scheme 1) as we previously described [21]. The key transformation of 2 with compound 5 consisted of four steps: (1) formation of ethanone 2; (2) tosylation of isopropylideneglycerol 3; (3) deprotection of isopropylidene ketal 4; and (4) ketal formation to generate 6. Compound 2 was prepared by reacting different kinds of commercially available a-bromoketone 1 with triazole in DMF using a method that we described previously [23]. The alkylation of isopropylidene glycerol 3 was achieved using a method as we described previously [22], and hydrolysis with $1 \mathrm{M} \mathrm{HCl}$ in $\mathrm{MeOH}$ yielded glyceryl tosylate 5. Ketal formation to generate 6 was carried out using 3 equivalents of trifluoromethane- sulfonic acid (TfOH) in toluene at room temperature for 60 hours, according to a method previously described [19]. All of the compounds synthesized in this work consist of four stereoisomers, and they were subjected to biological studies without further purification.

The fluorescent spectra of YCZ-FL and the fluorescent chromophore starting material 6-hydroxycoumarin were shown in Fig. 2. We found that YCZ-FL with a maximum exciting wavelength at $336 \mathrm{~nm}$ and a maximum emission wavelength at $442 \mathrm{~nm}$, respectively (Fig. 2). Comparing the maximum excitation and emission wavelength of 6-hydroxycoumarin, we found that there is a red band shift of the maximum excitation wavelength from $324 \mathrm{~nm}$ to $336 \mathrm{~nm}$, while a blue band shift of the maximum emission wavelength from $456 \mathrm{~nm}$ to $442 \mathrm{~nm}$ were observed. Interestingly, the fluorescence intensity of YCZ-FL was approximately 100 times to that of 6-hydroxycoumarin. This result indicates that
YCZ-FL exhibits a high fluorescent intensity which suggests that YCZ-FL is a effective fluorescent probe. This property is useful for application use of YCZ-FL on biological studies.
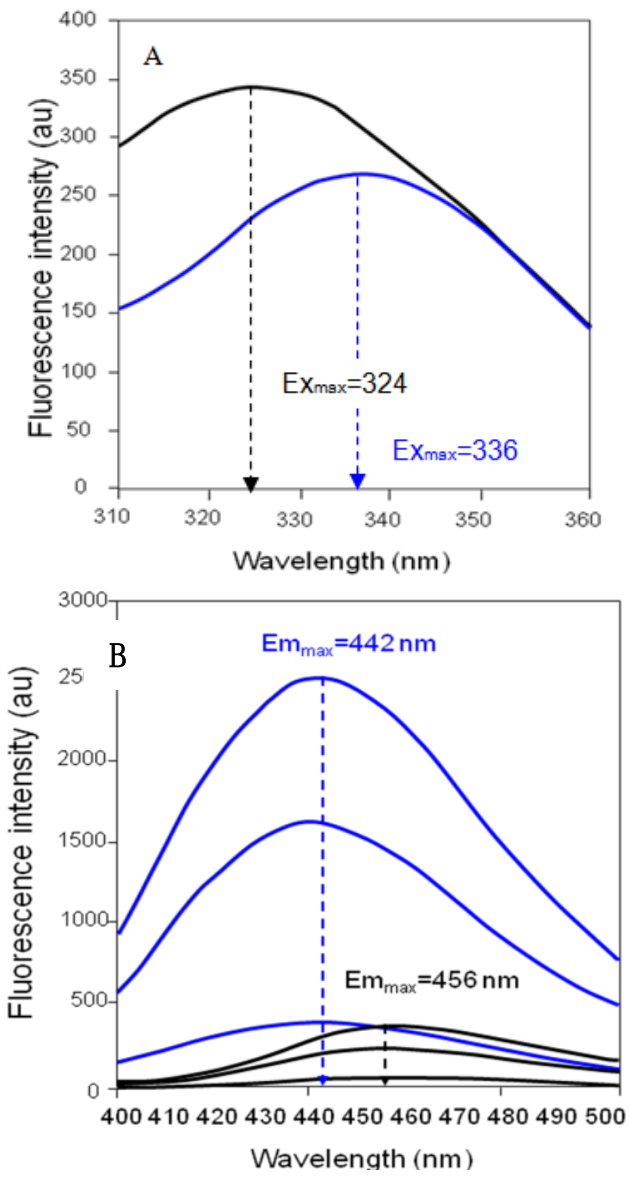

Fig. 2. Fluorescence spectra of 6-hydorxycoumarin and YCZ-FL.

The fluorescence spectra were recorded under a condition as described in the experimental section. Data shown in black is 6-hydorxycoumarin and in blue is YCZ-FL. (A) Excitation spectrum of 6-hydorxycoumarin $(100 \mu \mathrm{M})$ and YCZ-FL $(1 \mu \mathrm{M})$ were shown. (B) Emission spectrum of 6-hydorxycoumarin

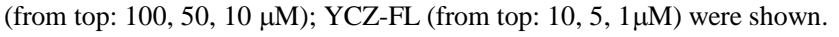

\section{B. Biology}

The bioassay used for determination the biological activity of YCZ-FL on BRs biosynthesis inhibition was carried out by a method as we described previously [19]. Arabidopsis BRs synthesis-deficient mutants such as dwarf 1 show remarkable dwarfism and the opening of the apical hook of cotyledons in the dark [7]. This unique de-etiolation in the dark phenotype has been used for screening for BR biosynthesis inhibitors [23]. With this assay system, we evaluated the biological activities of YCZ-FL.

To determine the biological activities of YCZ-FL on BRs biosynthesis inhibition, the concentrations of YCZ-FL were assigned to be $0,0.05,0.1,0.5,1,10$ and $50 \mu \mathrm{M}$. As shown in Fig. 3, the inhibition of stem elongation of the Arabidopsis seedlings by YCZ-FL was increasing with the increase of the concentration of YCZ-FL, and the $\mathrm{IC}_{50}$ value was found to be approximately $1.56 \pm 0.05 \mu \mathrm{M}$. In our previous work, an analogue of YCZ with naphthalene moiety (YCZ-25, the structure is shown in fig 1) exhibited inhibitory activity on retarding hypocotyls elongation of Arabidopsis seedling grown in the dark, with an $\mathrm{IC}_{50}$ value of $0.46 \pm 0.04$ $\mu$ M. [21] Although data obtained in the present work 
indicates that introduce a coumarin moiety instead of naphthalene weaken the biological activity. At a concentration of $10 \mu \mathrm{M}$, YCZ-FL can effectively inhibit stem elongation of Arabidopsis grown in the darkness. This observation clearly indicates that FCZ-FL is a useful fluorescent probe to determine the action mechanism of $\mathrm{YCZ}$ on BRs biosynthesis.

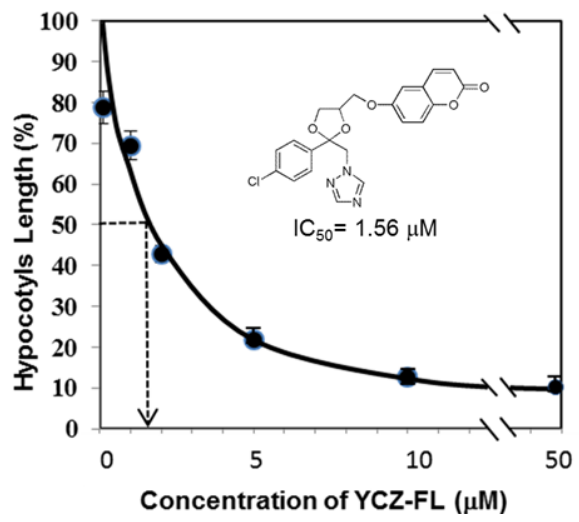

Fig. 3. Effect of YCZ-FL on retardation Arabidopsis seedlings grown in the darkness.

Arabidopsis seedlings were sown on a $1 \%$ solidified agar medium containing half Murashige and Skoog salt and grown in the darkness for 5 days as indicated in the experimental section. The final concentrations of YCZ-FL assigned to be $0,0.05,0.1,0.5,1,10$ and $50 \mu \mathrm{M}$. Average hypocotyl length at day 5 of treatment $(n>15)$. Error bars represent standard deviation. Experiments were duplicated to establish repeatability.

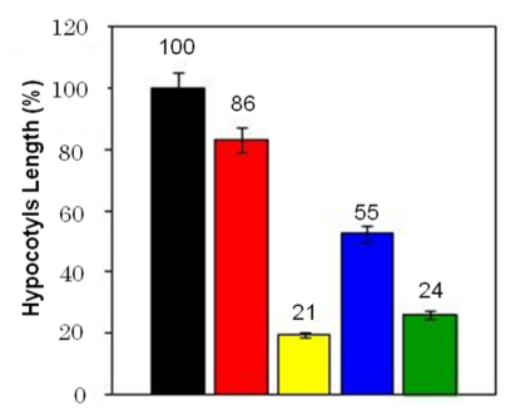

Fig. 4. Responses of Arabidopsis seedlings to inhibitor treatments and BL and GA complementation.

Arabidopsis seedlings were sown on a $1 \%$ solidified agar medium containing half Murashige and Skoog salt and grown in the darkness for 5 days as indicated in the experimental section. From left: control; co-application of $10 \mu \mathrm{M}$ 6-hydroxycoumarin; $10 \mu \mathrm{M}$ of YCZ-FL; $10 \mu \mathrm{M}$ of YCZ-FL with co-application of $10 \mathrm{nM}$ BL; $10 \mu \mathrm{M}$ of YCZ-FL with co-application of $1 \mu \mathrm{M} \mathrm{GA}_{3}$. Experiments were duplicated to establish repeatability.

It is known that GA biosynthesis inhibitors, such as paclobutrazol, retards the stem elongation of many plant species by blocking ent-kaurene oxidation and also mildly affect other cytochrome P450 mono-oxygenase [24]. This retardation can be rescued by the application of GA. In order to rule out the GA biosynthesis inhibition, we tested the effects of brassinolide (BL), the most biologically active BR, and GA on the recovery of chemical induced dwarfism of Arabidopsis seedlings grown in the dark. YCZ-FL was subjected to the bioassay at a concentration of $10 \mu \mathrm{M}$ and Arabidopsis seedlings were grown in the presence of BL (10 $\mathrm{nM})$ or GA $(1 \mu \mathrm{M})$ for 5 days in the darkness. Data were expressed in percentage by the comparison of none chemical treated control. As shown in Figure 4, YCZ-FL $(10 \mu \mathrm{M})$ exhibited high inhibitory activity on Arabidopsis seedlings elongation. The hypocotyls length was approximately $21 \pm$ $1 \%$ of none chemical treated seedlings. In the presence of 6-hydorxycoumarin $(10 \mu \mathrm{M})$, the plant height of Arabidopsis seedlings were approximately $86 \pm 3 \%$ of that of none chemical treated seedlings. This result indicates that 6-hydorxycoumarin may slightly inhibit the stem elongation of the Arabidopsis seedlings. Co-application of BL (10 nM) to YCZ-FL treated Arabidopsis seedlings showed recovery activity on stem elongation. The recovery degree is from $20 \pm$ $1 \%$ to $55 \pm 2 \%$. Co-application of GA $(1 \mu \mathrm{M})$ did not show significant recovery for YCZ-FL treated Arabidopsis seedlings (from 20 to $24 \%$ ). These results clearly indicate that YCZ-FL inhibits BRs biosynthesis in Arabidopsis seedlings. We expect further experimental use of YCZ-FL on dissecting the inhibition mechanism of YCZ will provide insight information about the regulation mechanism of BRs biosynthesis.

\section{CONCLUSIONS}

We reported the synthesis of 6-[2-(4-chlorophenyl)-2- [1, 2, 4] triazol-1-ylmethyl-[1, 3] dioxolan-4-ylmethoxy] coumarin (YCZ-FL) as a fluorescent probe of BRs biosynthesis inhibitors. Spectral analysis of YCZ-FL indicated that YCZ-FL exhibits strong fluorescence intensity which is approximately 100 times of that of 6-hydorxycoumarin. The biological activity of YCZ-FL on retardation of stem elongation of Arabidopsis seedlings indicated that YCZ-FL is a potent inhibitor with an $\mathrm{IC}_{50}$ value approximately $1.56 \pm 0.05 \mu \mathrm{M}$. To distinguish the primary site of actions of YCZ-FL on biosynthesis of GA and BRs, effect of co-application of GA $(1 \mu \mathrm{M})$ and/or BL (10 nM) on YCZ-FL treated Arabidopsis seedlings were investigated. We found co-application of BL reversed the inhibition of stem elongation treated by YCZ-FL, while GA did not. We expect further application use of YCZ-FL on determination the action mechanism of BRs biosynthesis inhibition may provide novel information about the regulation mechanism of plant growth and development.

\section{REFERENCES}

[1] S. D. Clouse and J. M. Sasse, "Brassinosteroids: Essential regulators of plant growth and development," Annu. Rev. Plant Physiol. Plant Mol. Biol., vol. 49, pp. 427-451, Jun. 1998.

[2] T. Altmann, "Molecular physiology of brassinosteroids revealed by the analysis of mutants," Planta, vol. 208, pp. 1-11, Mar. 1999.

[3] M. Nakaya, H. Tsukaya, N. Murakami, and M. Kato, "Brassinosteroids control the proliferation of leaf cells of Arabidopsis thaliana," Plant Cell Physiol, vol. 43, pp. 239-244, Feb. 2002.

[4] N. B. Mandava, "Plant growth-promoting brassinosteroids," Annu. Rev. Plant Physiol. Plant Mol. Biol., vol. 39, pp. 23-52, June 1988.

[5] J. Sasse, "Physiological actions of brassinosteroids: an update," J. Plant Growth Regul., vol. 22, pp. 276-288, Dec. 2003.

[6] S. Choe, S. Fujioka, T. Noguchi, S. Takatsuto, S. Yoshida, and K. A. Feldmann, "Overexpression of DWARF4 in the brassinosteroid biosynthetic pathway results in increased vegetative growth and seed yield in Arabidopsis," Plant J. vol. 26, pp. 573-582, June 2001.

[7] S. Choe, B. P. Dilkes, B. D. Gregory, A. S. Ross, H. Yuan, T. Noguchi, S. Fujioka, S. Takatsuto, A. Tanaka, S. Yoshida, F. E. Tax, and K. A. Feldmann, "The Arabidopsis dwarf1 mutant is defective in the conversion of 24-methylenecholesterol to campesterol in brassinosteroid biosynthesis," Plant Physiol., vol. 119, pp. 897-907, Mar. 1999. 
[8] G. J. Bishop, T. Nomura, T. Yokota, K. Harrison, T. Noguchi, S. Fujioka, S. Takatsuto, J. D. Jones, and Y. Kamiya, "The tomato DWARF enzyme catalyses C-6 oxidation in brassinosteroid biosynthesis," Proc. Natl. Acad. Sci., USA, vol. 96, pp. 1761-1766, Feb. 1999.

[9] Z. Hong, M. Ueguchi-Tanaka, K. Umemura, S. Uozu, S. Fujioka, S. Takatsuto, S. Yoshida, M. Ashikari, H. Kitano, and M. A. Matsuoka, "Rice brassinosteroid-deficient mutant, ebisu dwarf (d2), is caused by a loss of function of a new member of cytochrome P450," Pant Cell, vol. 15, pp. 2900-2910, Dec. 2003.

[10] T. Nomura, M. Nakayama, J. B. Reid, Y. Takeuchi, and T. Yokota, "Blockage of brassinosteroid biosynthesis and sensitivity causes dwarfism in garden pea," Plant Physiol., vol. 113, pp. 31-37, Jan. 1997 ,

[11] T. Sakamoto, Y. Morinaka, T. Ohnishi, H. Sunohara, S. Fujioka, M. Ueguchi-Tanaka, M. Mizutani, K. Sakata, S. Takatsuto, S. Yoshida, H. Tanaka, H. Kitano, and M. Matsuoka, "Erect leaves caused by brassinosteroid deficiency increase biomass production and grain yield in rice," Nat. Biotechnol., 2006, vol.. 24, pp. 105-109.

[12] Z. Y. Wang, T. Nakano, J. Gendron, J. He, M. Chen, D. Vafeados, Y. Yang, S. Fujioka, S. Yoshida, T. Asami, and J. Chory, "Nuclear-localized BZR1 mediates brassinosteroid-induced growth and feedback suppression of brassinosteroid biosynthesis," Dev. Cell, vol. 4, pp. 505-513, Apr. 2002.

[13] S. Hagihara, A. Miyazak, I. Matsuo, A. Tatami, T. Suzuki, and Y. Ito, "Fluorescently labeled inhibitor for profiling cytoplasmic peptide:N-glycanase," Glycobiology, vol. 10, pp. 1070-1076, Oct. 2007.

[14] K. Oh, "Jasmonic acid biosynthesis inhibitor," Regul. Plant Growth Devel. vol. 44, pp. 166-169, Apr. 2009.

[15] K. Oh and N. Murofushi, "Design and synthesis of novel imidazole derivatives as potent inhibitors of allene oxide synthase (CYP74)," Bioorg. Med. Chem, vol. 10, pp. 3707-3711, Dec. 2002.

[16] K. Oh, T. Asami, K. Matsui, G. A. Howe, and N. Murofushi, "Characterization of novel imidazole derivative, JM-8686, a potent inhibitor of allene oxide synthase," FEBS Lett., vol. 580, pp. 5791-5796, Oct. 2006.

[17] K. Oh, Y. Shimura, K. Ishikawa, Y. Ito, T. Asami, N. Murofushi, and Y. Yoshizawa, "Asymmetric synthesis and stereochemical structure-activity relationship of $(R)$ - and $(S)-8$-[1-(2,4-dichlorophenyl)-2-imidazol-1-yl-ethoxy] octanoic acid heptyl ester, a potent inhibitor of allene oxide synthase," Bioorg. Med. Chem., vol. 16, pp. 1090-1095, Feb. 2008.

[18] K. Oh, Y. Seki, N. Murofushi, and Y. Yoshizawa, "Effect of miconazole, an antifungal agent, on allene oxide synthase: Inhibition, kinetics, and binding," Pestic. Biochem. Physiol., vol. 94, pp. 107-111, Apr. 2009.

[19] K. Oh, K. Yamada, T. Asami, and Y. Yoshizawa, "Synthesis of novel brassinosteroid biosynthesis inhibitors based on the ketoconazole scaffold," Bioorg. Med. Chem. Lett., vol. 22, pp.1625-1628, Feb. 2012.

[20] K. Oh, K. Yamada, and Y. Yoshizawa, "Asymmetric synthesis and effect of absolute stereochemistry of YCZ-2013, a brassinosteroid biosynthesis inhibitor," Bioorg Med Chem Lett., vol. 23, pp. 6915-6919, Dec. 2013

[21] K. Yamada Y. Yoshizawa, and K. Oh, "Synthesis of 2RS,4RS-1-[2-phenyl-4-[2-(2-trifluromethoxy-phenoxy)-ethyl]-1,3-di oxolan-2-yl-methyl]-1H-1,2,4-triazole derivatives as potent inhibitors of brassinosteroid biosynthesis," Molecules, vol. 17, pp. 4460-4473, Apr. 2012

[22] K. Yamada, O. Yajima, Y. Yoshizawa, and K. Oh, "Synthesis and biological evaluation of novel azole derivatives as selective potent inhibitors of brassinosteroid biosynthesis," Bioorg. Med. Chem., vol. 21, pp. 2451-2461, May 2013.
[23] K. Sekimata, T. Ohnishi, M. Mizutani,Y. Todoroki, S. Y. Han, J. Uzawa, S. Fujioka, K. Yoneyama, Y. Takeuchi, S. Takatsuto, K. Sakata, S. Yoshida, and T. Asami, "Brz220 interacts with DWF4, a cytochrome $\mathrm{P} 450$ monooxygenase in brassinosteroid biosynthesis, and exerts biological activity," Biosci. Biotechnol. Biochem,. vol. 72, pp. 7-12, Jan. 2008.

[24] K. Sekimata, S. Y. Han, K. Yoneyama, Y. Takeuchi, S. Yoshida, and T. Asami, "A specific and potent inhibitor of brassinosteroid biosynthesis possessing a dioxolane ring," J. Agri. Food Chem., vol. 50, pp. 3486-3490, June 2002

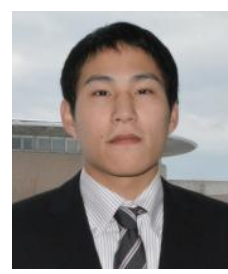

Tomoki Hoshi was born in Fukushima Prefecture, Japan. He is currently a graduate student of the graduate school of Akita Prefectural University. His research interests are chemical design and synthesis biological active chemicals.

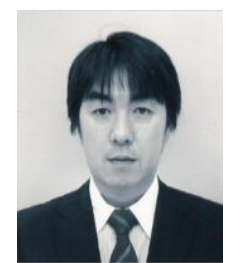

Tadashi Matsumoto was born in Akita Prefectural, Japan. He received his Ph.D. degree from Akita Prefectural University in 2009. He is currently serving as Research Associate at the Department of Biotechnology, Akita Prefectural University, Japan His research interests include biochemistry and molecular biology of plants.

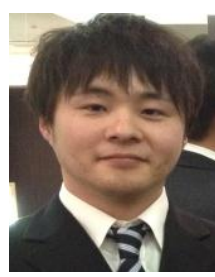

Kazuhiro Yamada was born in Nigata Prefectural, Japan. He received his master's degree from Department of Biotechnology, Akita Prefectural University. He is currently serving as a researcher at Bushu Pharmaceuticals Ltd. His research interests are chemical synthesis biological active compounds.

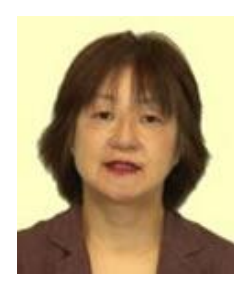

Yuko Yoshizawa was born in Tokyo, Japan. She received his Ph.D. degree from Hokkaido University. She is currently serving as a professor of bioorganic chemistry at the Department of Biotechnology, Akita Prefectural University, Japan. Her research interests identification of natural products from plant sources.

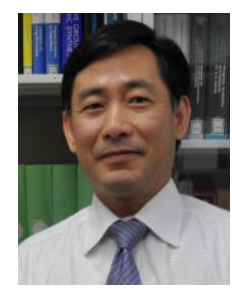

Keimei Oh was born in Shanghai, China and received his Ph.D. degree from the University of Tokyo in 1997. He is currently serving as an associate professor at the Department of Biotechnology, Akita Prefectural University, Japan. His research interests are the design and synthesis specific inhibitors of plant hormone biosynthesis and study the mode of action of bio-active chemicals. 